\title{
Low-Temperature Electron Mobility in Parabolic Quantum Wells
}

\author{
R. M. Seraide and G.-Q. Hai \\ Instituto Física de São Carlos, USP, 13566-590 São Carlos, SP, Brazil
}

Received on 23 April, 2001

\begin{abstract}
We present a theoretical study on the electron mobility and scattering mechanism in a remotely doped AlGaAs wide parabolic quantum well. Electron mobilities in different subbands are calculated from the self-consistent results of the subband energy and wavefunction in the system. The scattering due to ionized impurities and alloy disorder is considered. We show the interplay of the different scattering mechanisms.
\end{abstract}

\section{Introduction}

Improvements of the semiconductor growth techniques have offered the possibility to obtain low-dimensional semiconductor structures with desired well shapes. One of those structures is the so-called parabolic quantum well (PQW). The PQW's based on GaAs have been developed by tailoring the conduction-band edge of a graded $\mathrm{Al}_{x} \mathrm{Ga}_{1-x} \mathrm{As}$ semiconductor ternary alloy through properly varying the Al mole fraction $x$.[1, 2] Because a thick and uniform electron slab (in order of $10^{3} \AA$ ) can be obtained in a wide PQW, remotely doped wide PQW's have been proposed as structures in which a high-mobility quasi-three-dimensional (Q3D) electron gas can be realized. Magneto-transport experiments on these systems confirmed the existence of a thick slab of high-mobility electron gas.[2]

Although a number of experimental and theoretical studies have been done on the electron transport properties in such a system since the first AlGaAs PQW was realized by MBE twelve years ago, the scattering mechanism and electron mobility are not well understood yet.[3] In fact, the so-called Q3D electron gas is a system composed by a wide Q2D system with multiple electron subbands populated. Clearly, the intersubband interaction is of important contribution to the electron transport. Furthermore, beyond the remotely doped impurity scattering, other scattering mechanisms can be decisive to the electron mobility such as alloy scatterng and background acceptors in a real sample.

In this work, we study theoretically the electron mobility and scattering mechanism in AlGaAs PQW's. We start with a self-consistent calculation of the subband structure in Sec. II which yields the subband energy and the wavefunction in the system. Two layers of donors are remotely doped asymmetrically in two sides of the well. Typically more than one subbands are populated. The ionized impurity scattering and alloy scattering are considered in Sec. III. The transport mobilty of electrons in different subbands are calculated by solving the Boltzmann equation. We also considered the effects of the background acceptors on the electron mobilities. We show the interplay of the different scattering mechanisms.

\section{Electronic structures}

The electron energy and wavefunction in a Q2D system can be written as $E_{n}(\vec{k})=E_{n}+\hbar^{2} k^{2} / 2 m^{*}$ and $\Psi_{n, \vec{k}}(x, y, z)=\psi_{n}(z) \exp (i \vec{k} \cdot \vec{r}) / \sqrt{A}$, where $n=1,2$, $\ldots$ is the subband index, $\vec{r}(\vec{k})$ the electron position (wavevector) in the $x y$-plane, $E_{n}$ the subband energy, $\psi_{n}(z)$ the electron wavefunction in the $z$-direction, $m^{*}$ the electron effective mass, and $A$ the area of sample.

The parabolic quantum wells are formed by $\mathrm{Al}_{0.3} \mathrm{Ga}_{0.7} \mathrm{As}$ barrier and the concentration of the $\mathrm{Al}$ is varied from $x=0$ at the center of the well to $x=0.1$ at the edge of the well. By tailoring the conductionband edge of $\mathrm{Al}_{x} \mathrm{Ga}_{1-x} \mathrm{As}$, we construct the following confinement potential to the electrons

$$
V_{C}(z)= \begin{cases}\frac{1}{2} m^{*} \Omega^{2} z^{2}, & |z| \leq W_{Q W} / 2 \\ V_{B}, & |z|>W_{Q W} / 2\end{cases}
$$

where $V_{B}$ is the potential of the barrier, $\Omega=$ $2 \sqrt{2 V_{b} / m^{*}} / W_{Q W}$, and $W_{Q W}$ is the width of the quantum well. In the above expression, the potential at the center of the QW is zero (GaAs). At the edges of the well $\left(z= \pm W_{Q W} / 2\right), V_{C}=V_{b}$. Notice that $V_{b} \leq V_{B}$. The conduction band off-set between $\mathrm{Al}_{x} \mathrm{Ga}_{1-x} \mathrm{As}$ and GaAs is a function of $x$ given by $V(x)=0.6 \times\left(1.155 x+0.037 x^{2}\right) \mathrm{eV}$. In the present calculation, we take $V_{B}=228 \mathrm{meV}(x=0.3)$ for $\mathrm{Al}_{0.3} \mathrm{Ga}_{0.7} \mathrm{As}$ barrier and $V_{b}=72 \mathrm{meV}(x=0.1)$. The 
system is remotely doped with two layers of impurities in two sides of the quantum well. The impurity distribution is taken the following form

$$
n_{D}(z)= \begin{cases}N_{D, L} / W_{D, L}, & \left|z-Z_{D, L}\right| \leq W_{D, L} / 2 \\ N_{D, R} / W_{D, R}, & \left|z-Z_{D, R}\right|<W_{D, R} / 2 \\ 0, & \text { otherwise }\end{cases}
$$

where $N_{D, L}, W_{D, L}$ and $Z_{D, L}\left(N_{D, R}, W_{D, R}\right.$ and $\left.Z_{D, R}\right)$ are the areal concentration, the the thickness and the position of the doped impurity layer on the left (right) side of the quantum well, respectively.

The subband energy $E_{n}$ and the electron wavefunction $\psi_{n}(z)$ are deterimed by the Schrödinger equation in the $z$-direction. The effective confinement potential entering the Hamiltonian is $V_{e f f}(z)=V_{H}(z)+V_{C}(z)$ which is composed as a sum of the potential $V_{C}(z)$ given in Eq. (1) and the Hartree potential $V_{H}(z)$. The Hartree potential, governed by the Poisson equation, dependes on the ionized imprity and electron distribution $n_{e}(z)$. At zero-temperature, the electron distribution is given by

$$
n_{e}(z)=\sum_{n=1}^{N}\left|\psi_{n}(z)\right|^{2} \int_{E_{n}}^{E_{F}} \rho(E) d E
$$

where $N$ is the number of the occupied subbands, $\rho(E)$ is the electron density of states of the system and $E_{F}$ is the Fermi energy. Because the electron distribution depends on $E_{n}$ and $\psi_{n}(z)$, the solution of the Poisson equation is, in turn, dependent on the Schrödinger equation. In this work, we solve the coupled Schrödinger and Poisson equations selfconsistently. In the calculation, the total areal electron density $N_{e}=\int_{-\infty}^{\infty} n_{e}(z) d z$ is determined by the difference between $N_{D}$ and $N_{A}$, where $N_{D}=N_{D, L}+N_{D, R}$ is total areal donor concentrations and $N_{A}$ is the total areal acceptor concentrations. $N_{A}$ can be estimated from the thickness of the depletion layer with a uniform distribution of the background acceptors $n_{A}$. Fig. 1 gives an example of the self-consistent solutions for a quantum well of width $W_{Q W}=760 \AA$. The effective confinement potential profile $V_{e f f}(z)$ (thicksolid curve), the first three quantized electron levels $E_{n}$ and the corresponding wavefunctions $\psi_{n}(z)$ are demonstrated. In the figure, the energy is measured from the Fermi energy $E_{F}$. Two donor impurity layers are $200 \AA$ away from the right and left edge of the quantum well indicated by the verticle dotted lines. The background acceptor concentration is taken as $n_{A}=10^{15} \mathrm{~cm}^{-3}$.

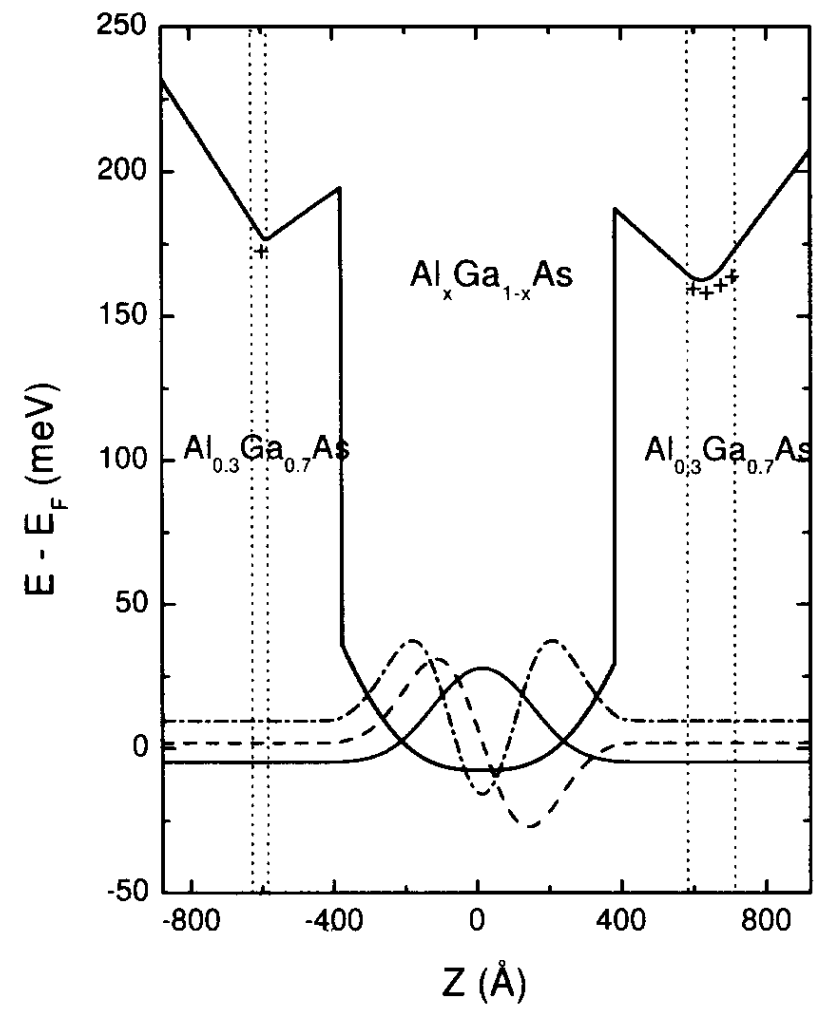

Figure 1. The electronic structure of a parabolic AlGaAs quantum well of width $760 \AA$. The donor distribution is defined by the parameters: $N_{D, L}=N_{D, R}=1.5 \times 10^{12}$ $\mathrm{cm}^{-2}, W_{D, L}=20 \AA, W_{D, R}=100 \AA, Z_{D, L}=-590 \AA$, and $Z_{D, R}=630 \AA$. The acceptor concentration is $n_{A}=10^{15}$ $\mathrm{cm}^{-3}$. The first three levels $E_{n}$ are indicated and the corresponding wavefunctions are given by solid, dash, and dashdotted curves. The vertical thin-dotted lines and the symbles "+" indicate the two dopping layers.

\section{Scattering mechanism and electron mobility}

At low-temperature, the most possible scattering mechanisms limitting the electron mobility in the present system are the remotely doped ionized donors, the alloy disorder potential of AlGaAs, and the background acceptors.[4, 5]

The screening of the electron gas is essential to determine the scattering due to ionized impurities. The screened ionized impurity potential can be obtained in terms of the static dielectric response function within the random-phase approximation (RPA). Because of the occupation of several subbands, the dielectric function has a tensor character given by $\epsilon_{n n^{\prime}, m m^{\prime}}(\vec{q})$. If we assume that the impurities are uniformly distributed in the $x y$-plane and are uncorrelated, the transition matrix element due to the screened Coulomb scattering potential is given by,[4]

$$
\left|M_{n n^{\prime}}(\vec{q})\right|^{2}=\left(\frac{2 \pi e^{2}}{\epsilon_{0} q}\right)^{2} \int d z_{i} n_{\mathrm{imp}}\left(z_{i}\right)\left|u_{n n^{\prime}}(q)\right|^{2},
$$


with

$$
u_{n n^{\prime}}(q)=\sum_{m m^{\prime}} \epsilon_{n n^{\prime}, m m^{\prime}}^{-1}(\vec{q}) G_{m m^{\prime}}\left(q, z_{i}\right)
$$

and

$$
G_{m m^{\prime}}\left(q, z_{i}\right)=\int d z \psi_{m}(z) \psi_{m^{\prime}}(z) e^{-q\left|z-z_{i}\right|},
$$

where $\vec{q}=\vec{k}-\vec{k}^{\prime}$ is the change in electron momentum due to scattering. In equation (2), we take $n_{\mathrm{imp}}(z)=n_{D}(z)$ for remotely doped donor scatteing and $n_{\operatorname{imp}}(z)=n_{A}$ for background acceptor scattering. by $[6]$

For alloy scattering, the scattering matrix is given

$$
\left|M_{n n^{\prime}}(\vec{q})\right|^{2}=(\delta V)^{2} \int d z[1-x(z)] x(z)\left|\psi_{n}(z) \psi_{n^{\prime}}(z)\right|^{2},
$$

where $x(z)$ is the position dependent $\mathrm{Al}$ concentration. For AlGaAs, $\delta V=0.6 \mathrm{eV}$. Notice that, the matrix element due to alloy scattering is independent on $\vec{q}$.

Using the above scattering matrix elements due to different scattering mechanism, we calculate the electron transport mobility from the Boltzmann equation in the present multisubband Q2D system.[4]

Fig. 2 shows the transport mobilities as a function of total electron density for the electrons in subbands $n=1,2$, and 3 due to three different types of scattering. The numerical calculations are performed for the same structure as shown in Fig. 1 but now we increase the donor density $N_{D, L}$ and $N_{D, R}$ simultaneously. As a consequence, the electron density is increased. At low electron densities, the mobility of the electrons in the lowest subband $n=1$ increases with increasing the electron (also donor) density. This is mainly due to the screening effects and the increase of the kinetic energy of the conduction electrons on the Fermi surface. At the onset of the occupation of the second subband $n=2$, the abrup change of the mobility results from the interplay between the intersbband scattering and the intersubband coupling effect in the dielectric function (which enhances the screening and is more effective for small subband Fermi energy $E_{F}-E_{n}$ ). When there are two or three subbands occupied, the mobility decrease of the lower subbands is mainly induced by the intersubband scattering. For the considered background acceptor concentration $n_{A}=10^{15} \mathrm{~cm}^{-3}$, the acceptor scattering is the dominant mechanism to the electron transport. We also notice that the alloy scattering become more pronounced at high electron densities.

In conclusion, we have calculated the electron subband mobilities from the self-consistent electronic structure of the AlGaAs wide parabolic quantum wells. The numerical results shows that, for the considered structure with a background acceptor concentration $n_{A}=$ $10^{15} \mathrm{~cm}^{-3}$, the mobility is dominated by the scattering of these acceptors. Our results also indicate the importance of the intersubband scattering.

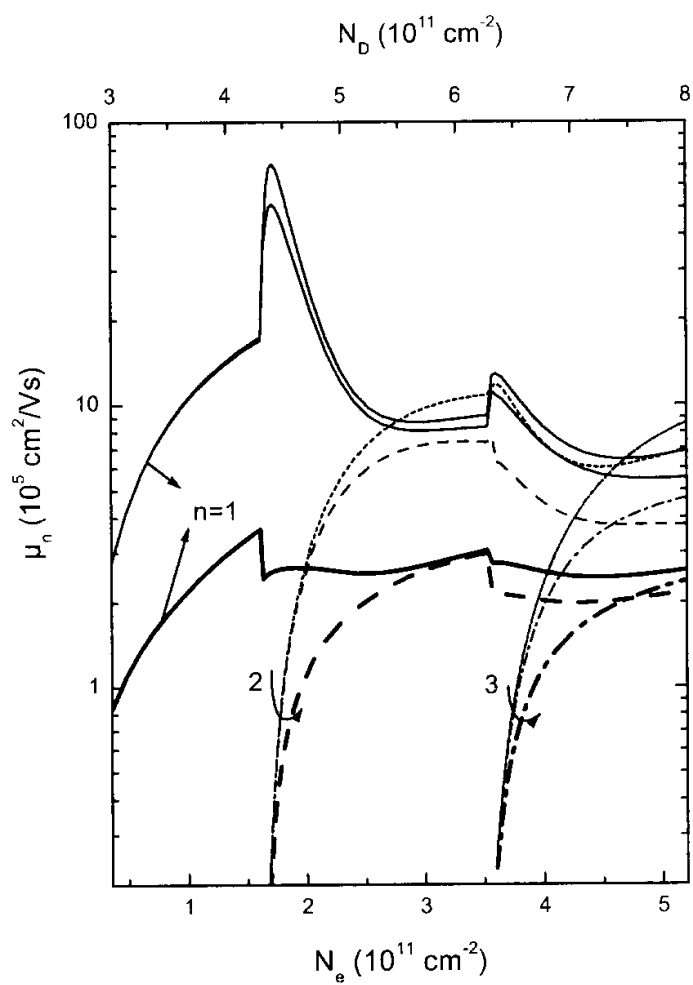

Figure 2. The transport mobility as a function of the total electron density for electrons in the first three subbands $n=1$ (solid curves), 2 (dash curves), and 3 (dash-dotted curves). The mobilities result from the ionized donor scattering only (thin-curves), the ionized donor + alloy scattering, and the ionized donor + acceptor + alloy scattering (the thick-curves) are plotted.

Acknowledgments This work was supported by FAPESP and CNPq (Brazil).

\section{References}

[1] E. G. Gwinn, R. M. Westervelt, P. F. Hopkins, A. J. Rimberg, M. Sundaram, and A. C. Gossard, Phys. Rev. B 39, 6260 (1989); T. Sajoto, J. Jo, M. Santos, and M. Shayegen, Appl. Phys. Lett. 55, 1430 (1989); K. Karrai, H. D. Drew, H. W. Lee, and Shayegan, Phys. Rev. B 39, 1426 (1989).

[2] A. Wixforth, Surf. Sci. 305, 194 (1994), and references therein; P. F. Hopkins, A. J. Rimberg, E. G. Gwinn, R. M. Westervelt, M. Sundaram, and A. C. Gossard, Appl. Phys. Lett. 57, 2823 (1990).

[3] G. Salis, P. Wirth, T. Heinzel, T. Ihn, K. Ensslin, K. Maranowski, and A. C. Goss ard, Phys. Rev. B 59 R5304 (1999), and references therein.

[4] G. Q. Hai, N. Studart, and F. M. Peeters, Phys. Rev. B 52, 8363 (1995); G. Q. Hai and N. Studart, Phys. Rev. B 55, 6708 (1997).

[5] G. Q. Hai and N. Studart, Phys. Rev. B 52, R2245 (1995).

[6] R. M. Seraide and G. Q. Hai, unpublished. 